\title{
Transient bacteraemia due to Treponema amylovorum in a Renal Transplant Patient
}

\author{
Amelie Morin ${ }^{\mathrm{a}}$, Solene Patrat-Delon ${ }^{\mathrm{b}}$, Martine Bonnaure-Mallet ${ }^{\mathrm{a}, \mathrm{c}}$, Sophie Gie ${ }^{\mathrm{d}}$, \\ Anne Jolivet-Gougeon ${ }^{\mathrm{a}, \mathrm{c}, \mathrm{e}}$
}

\begin{abstract}
Transient bacteraemia occurs frequently after dental procedures or daily life activities and may be etiologic factors in the development of cardiovascular diseases; the origin is the oral cavity, which is intensively colonized by bacteria. Treponema amylovorum is one of these oral bacteria, only isolated in endodontic lesions. We described the first case of a transient bacteraemia due to T. amylovorum in an immuno-compromised patient, isolated from the blood through $16 \mathrm{~S}$ rRNA gene sequencing. This case report highlights the probable underestimation of cases of bacteraemia due to oral Treponema species.
\end{abstract}

Keywords: Bacteraemia; Endodontic infection; 16S rRNA; Treponema; T. amylovorum

\section{Introduction}

Odontogenic bacteraemia is transient and well described; such bacteraemia occurs after dental procedure, but also after daily life activities; it may lead to distant site infections, and is associated with the development of cardiovascular disease [1]. Streptococci are the predominant organisms with Fusobacteria, Actinobacteria or Bacteroidetes [1]. Montagner et al [2] showed that Treponema species are implicated in acute endodontic infections, especially T. amylovorum isolated in $5 / 20$ patients with infected root canals and $9 / 20$ patients with

Manuscript accepted for publication September 24, 2012

${ }^{\text {a}}$ Pole Microorganismes, CHU Pontchaillou, Rennes, France

${ }^{\mathrm{b}}$ Service des Maladies Infectieuses, CHU Pontchaillou, Rennes, France

${ }^{c}$ Equipe Microbiologie EA 1254, Universite de Rennes 1, Universite

Europeenne de Bretagne, Rennes, France

${ }^{\mathrm{d}}$ Service de Nephrologie, CHU Pontchaillou, Rennes, France

${ }^{\mathrm{e}}$ Corresponding author: Anne Jolivet-Gougeon, Equipe

Microbiolo- gie, EA 1254, Faculte des Sciences Pharmaceutiques et

Biologiques, Universite de Rennes 1, 2 Avenue du Professeur Leon

Bernard, 35043 Rennes, France. Email: anne.gougeon@univ-rennes1.fr

doi: http://dx.doi.org/10.4021/jmc869w acute apical abscesses. Siqueira and Rocas [3] investigated occurrence of T. amylovorum and found it in 2 of 31 cases (7\%; 5\% asymptomatic and 10\% symptomatic). Currently, there are no data in the literature describing the presence of Spirochaetes inhabiting the oral cavity in blood.

Here we report the first case of an immuno-compromised patient who presented a bacteraemia due to Treponema amylovorum; then we discuss the interpretation of the presence of such a bacteria in blood.

\section{Case Report}

The patient is a 30 -year-old woman, followed in nephrology for 27 years; her renal history began in 1984 with a typical hemolytic uremic syndrome, leading to hemodialysis since 1987. She underwent three renal transplants (in 1989, 1995 and 2006), resulting in three rejections. The last one occurred in 2006, with graft dysfunction and return in hemodialysis on Canaud's catheter in August 2010, three times a week. The patient also presents a peripheral arterial disease. Her treatment included prednisone $5 \mathrm{mg}$ per day and tacrolimus $1.5 \mathrm{mg}$ per day, but she stopped by herself these drugs in February 2011.

Since March 8, 2011, she has complained of a left iliac fossa pain in the renal transplant area, with fever $\left(38{ }^{\circ} \mathrm{C}\right)$ without chills. Clinical examination just confirmed the pain, without contracture; the patient did not present any dental pain. One blood culture was sampled on March 10, and she was discharged from the hospital. The blood culture became positive after a 7 day incubation period and microbial diagnosis led to identify Treponema amylovorum.

On 15 March, the patient was admitted in the emergency unit for renal transplant's pain, and she received an antalgic treatment with morphine; the body temperature was $37.9^{\circ} \mathrm{C}$. Biological results showed an elevated $\mathrm{C}$ reactive protein $(86$ $\mathrm{mg} / \mathrm{L}$ ), a leucocytes count at $10.6 \mathrm{G} / \mathrm{L}$ with a majority of neutrophils, the presence of an anemia with hemoglobin at $9.2 \mathrm{~g} / \mathrm{dL}$, an elevated creatinine at $406 \mu \mathrm{mol} / \mathrm{L}$, urea at 10.6 $\mathrm{mmol} / \mathrm{L}$ and an elevated potassium rate at $5.9 \mathrm{mmol} / \mathrm{L}$. Two blood cultures, sampled on 17 and 18 March, remained negative after 5 days of incubation, the urine culture revealed $10^{6}$ 


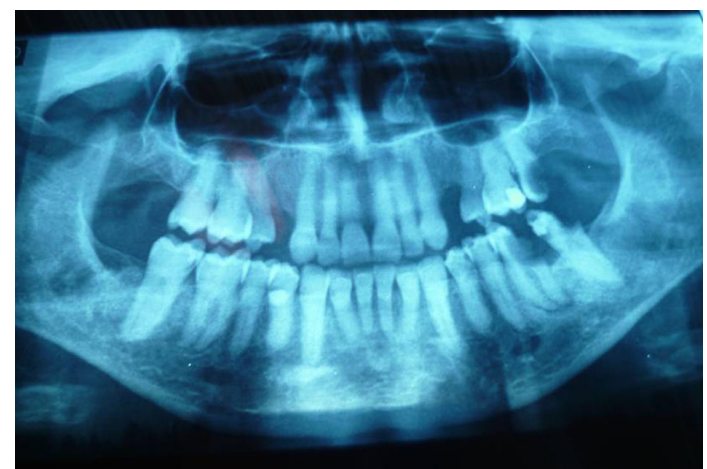

Figure 1. Dental panoramic showing periradicular infections on teeth 26-27-36 and 37.

leucocytes $/ \mathrm{mL}$ with a polymicrobial flora at $10^{4} / \mathrm{mL}$.

The bacteraemia was considered as a transient bacteraemia, from a dental origin. An oral cavity examination was realized on 10 May: three distal caries were identified on teeth 27,36 and 37 with endodontic infection. A dental panoramic showed a focus of periradicular infections (Fig. 1), requiring dental extractions of these three molars, under antibioprophylaxis, that were realized one month later.

So far, she never presented any symptoms in relation with this bacteraemia, apart the first febrile episode which has been associated to the transplant rejection. The valid diagnosis was transplant rejection, requiring the reintroduction of $20 \mathrm{mg} / \mathrm{d}$ of prednisone and transplantectomy on 1st April. The patient did not receive any antibiotic treatment, clinical outcome was favorable with apyrexia and cessation of pain.

\section{Microbiological identification}

In the laboratory, the diagnosis was made by blood cultures using the Bactec ${ }^{\circledR}$ vials (Plus Aerobic/F; Lytic/10 Anaerobic/F; Becton Dickinson) incubated 7 days at $35{ }^{\circ} \mathrm{C}$ in an incubator-rocker compartment. After incubation, direct microscopic examination revealed Gram negative helically shaped bacteria (7 microns long), suggestive of Trepone$m a$ genus. These strictly anaerobic, motile bacteria do not grow easily on commonly used media. When identification by conventional methods fails or in case of the suspicion of non-culturable bacteria, the molecular diagnosis can often achieve a result [3]. We used PCR amplification and sequencing of the rrs gene encoding $16 \mathrm{~S}$ ribosomal RNA. Sequences were compared to a database to allow specific identification (NCBI Blast: http://www.ncbi.nlm.nih.gov/ genome?term=blast, last accessed November 11, 2012).

\section{Discussion}

Transient bacteraemia, not exceeding 60 minutes after the procedure at risk, are well described after dental treatment procedures, and also after daily life activities such as eating, chewing or tooth brushing in individuals with periodontal inflammation [4]. Odontogenic bacteraemia certainly results from a breach in the oral mucosal barrier allowing the penetration of bacteria into the bloodstream from oral niches; besides, innate microbial factors may play a role, such as virulence factors that mediate tissue penetration and vascular invasion. Lockhart et al [4] inventoried in the literature 126 bacterial species reported in blood cultures following dental extractions or tooth brushing. These bacteraemia were characterized by low concentrations of bacteria in the blood and a rapid clearance of the bacteria by host defenses; those arguments could explain the difficulties to isolate new species or fastidious organisms.

The oral cavity is intensively colonized by bacteria (bacterial plaque from $10^{11}$ and $10^{12} \mathrm{CFU} / \mathrm{g}$ ). More than $700 \mathrm{bac}-$ terial species have been isolated in the mouth, and about 20 species are usually cultured on commonly used media. Dental abscess is usually polymicrobial, including facultative or strict anaerobes, and non cultivable bacteria as Treponema, detected with molecular techniques [5]. Oral Treponema species may represent more than $50 \%$ of the polymicrobial flora of the dental plaque $(70 \%$ of species are non cultivable), but less than $1 \%$ of the microorganisms in oral cavity of a healthy subject. T. denticola has been associated with periodontitis, with expression of a wide range of virulence factors that mediate tissue penetration, destruction and evasion of host immune responses [6]. T. denticola has also been detected in cardiovascular specimens (cardiac valves, aortic aneurysms), as well as others oral bacteria [7]. For T. amylovorum, few data are available on its pathogenicity, except in periodontal diseases; one study even reported no relationship between the presence of T. amylovorum in dental plaque and associated periodontitis [8].

Direct examination is highly evocative of a Treponema species, cultivation is often difficult, but recent advances in bacterial identification methods, such as 16S rRNA gene sequencing, showed the diversity of bacteria inhabiting the oral cavity $[3,9]$.

\section{Conclusion}

We describe the first isolation of T. amylovorum in a blood culture, in an immuno-compromised patient. This transient bacteraemia is thought to come from oral origin, with no immediate severe clinical consequence. This case report highlights the probable underestimation of cases of bacteraemia due to oral Treponema species. Recently, a statistically significant association of oral invasive Treponema with Alzheimer's disease has been postulated, using detection of species specific PCR and antibodies in the brains of these patients [10]. Early detection of periodontal pathogens could be useful to better prevent these various diseases, especially in patients with risk factors. 


\section{Acknowledgement}

We thank Philippe Gautier for technical assistance. The authors deny any conflicts of interest related to this study.

\section{Conflict of Interest}

None.

\section{Declaration of Funding}

None.

\section{References}

1. Parahitiyawa NB, Jin LJ, Leung WK, Yam WC, Samaranayake LP. Microbiology of odontogenic bacteremia: beyond endocarditis. Clin Microbiol Rev. 2009;22(1):46-64, Table of Contents.

2. Montagner F, Jacinto RC, Signoretti FG, Gomes BP. Treponema species detected in infected root canals and acute apical abscess exudates. J Endod. 2010;36(11):1796-1799.

3. Siqueira JF, Jr., Rocas IN. PCR-based identification of Treponema maltophilum, T amylovorum, T medium, and Tlecithinolyticum in primary root canal infections. Arch
Oral Biol. 2003;48(7):495-502.

4. Lockhart PB, Brennan MT, Sasser HC, Fox PC, Paster BJ, Bahrani-Mougeot FK. Bacteremia associated with toothbrushing and dental extraction. Circulation. 2008;117(24):3118-3125.

5. Bahrani-Mougeot FK, Paster BJ, Coleman S, Ashar J, Knost S, Sautter RL, Lockhart PB. Identification of oral bacteria in blood cultures by conventional versus molecular methods. Oral Surg Oral Med Oral Pathol Oral Radiol Endod. 2008;105(6):720-724.

6. Visser MB, Ellen RP. New insights into the emerging role of oral spirochaetes in periodontal disease. Clin Microbiol Infect. 2011;17(4): 502-512.

7. Nakano K, Nemoto H, Nomura R, Inaba H, Yoshioka H, Taniguchi K, Amano A, et al. Detection of oral bacteria in cardiovascular specimens. Oral Microbiol Immunol. 2009;24(1):64-68.

8. Willis SG, Smith KS, Dunn VL, Gapter LA, Riviere KH, Riviere GR. Identification of seven Treponema species in health- and disease-associated dental plaque by nested PCR. J Clin Microbiol. 1999;37(3):867-869.

9. Wyss C, Choi BK, Schupbach P, Guggenheim B, Gobel UB. Treponema amylovorum sp. nov., a saccharolytic spirochete of medium size isolated from an advanced human periodontal lesion. Int J Syst Bacteriol. 1997;47(3):842-845.

10. Miklossy J. Alzheimer's disease - a neurospirochetosis. Analysis of the evidence following Koch's and Hill's criteria. J Neuroinflammation. 2011;8:90. 